\title{
marges Marges
}

revue d'art contemporain Revue d'art contemporain

\section{Walid Raad, « Scratching on Things I Could Disavow: A History of Art in the Arab World »}

Paris, 104, 6 novembre - 5 décembre 2010

Irène Panzani

\section{OpenEdition}

\section{Journals}

Édition électronique

URL : http://journals.openedition.org/marges/373

DOI : $10.4000 /$ marges.373

ISSN : 2416-8742

\section{Éditeur}

Presses universitaires de Vincennes

Édition imprimée

Date de publication : 15 octobre 2012

Pagination : 166-167

ISBN : 978-2-84292-354-9

ISSN : $1767-7114$

\section{Référence électronique}

Irène Panzani, «Walid Raad, « Scratching on Things I Could Disavow: A History of Art in the Arab

World » », Marges [En ligne], 15 | 2012, mis en ligne le 15 octobre 2013, consulté le 22 septembre 2020. URL : http://journals.openedition.org/marges/373 ; DOI : https://doi.org/10.4000/marges.373 


\section{Walid Raad, « Scratching on Things I Could Disavow: A History of Art in the Arab World 》}

Paris, 104, 6 novembre -5 décembre 2010

Walid Raad, artiste en résidence au 104 en 2010, prend comme objet de travail le lien entre art et histoire. Depuis des années, il interroge par des moyens visuels la situation politique et sociale de son pays, le Liban, tout en le situant dans le contexte complexe du Moyen-Orient. À partir de documents comme des photographies, des vidéos, des notes, Raad construit des récits qui s'inspirent de la réalité. Il transforme les faits en images, les statistiques en taches colorées. Ces éléments sont récoltés ou encore produits par l'artiste même. Ainsi, le spectateur se retrouve face à une sorte de fiction documentaire, un archivage de métaphores capable d'exprimer la réalité, quelque fois mieux que les médias.

En entrant dans l'Atelier 4 du 104, le public se trouve en face d'une porte ou plutôt, de son seul cadre de bois. Une carte projetée sur le mur de l'entrée interroge le regard et se montre dans sa complexité. Son contenu se réfère au développement de nouvelles infrastructures et de bases de données dédiées aux arts visuels au Moyen-Orient. C'est autour de cette projection que débute la performance. Les spectateurs sont invités à s'asseoir sur des tabourets, comme un groupe d'enfants attendant le conteur qui doit arriver. À travers la voix de Carlos Chahine, Walid Raad met en évidence les enjeux et problématiques de l'implantation du monde de l'art contemporain à Abou Dhabi, au Qatar, et ailleurs dans le Golfe Persique. II réfléchit en particulier à l'impossible communication entre ce monde et la population locale. Qui de cette population pourra se rendre au Musée Guggenheim et dans les autres infrastructures culturelles en construction sur l'île de Saadiyat? Qui aura accès à cette tour d'ivoire? L'encadrement de la porte précédemment évoqué devient donc 
une métaphore du gouffre existant entre ces hétérotopies occidentales et le quotidien des habitants du lieu.

Ensuite, le spectateur se lève et suit le conteur au-delà de la porte, s'arrêtant en face d'une autre œuvre: une maquette miniature d'une galerie ou d'un musée. II s'agit de The Atlas Group (1989-004), pièce créée à l'occasion d'une exposition à la galerie Sfeir Semler à Beyrouth. L'artiste avait refusé d'y montrer son travail en 2005 et longtemps après, cette œuvre est d'une certaine façon l'explication de ce refus. Dans un entretien réalisé par Hélène Chouteau, il affirme: «j'avais l'impression que les clichés, les cassettes vidéo, les textes et les sculptures qui constituaient l'Atlas Group Archive avaient été affectés d'une façon étrange; je sentais qu'au moment où les œuvres seraient présentées dans la galerie (si elles l'étaient), elles apparaîtraient à mes yeux et peut-être à ceux des autres, au 1/100e de leur échelle originale. J'étais convaincu que les œuvres rétréciraient une fois exposées à Beyrouth. Ou plutôt [...], que pour que ces œuvres apparaissent à leur bonne échelle, il faudrait d'abord que je les réduise au $1 / 100^{\mathrm{e}}$ de leur échelle originale ». La maquette exemplifie le changement de poids du travail de l'artiste dans son pays et, peut-être, son impuissance vis-à-vis de la situation historique libanaise.

Enfin, le spectateur se retrouve face à un fragment de mur, fragment qui fait penser à une ruine de guerre, mais aussi à une cimaise coupée de son lieu d'origine. Les deux cotés du mur sont habillés de listes de noms écrits sur des supports de formes et couleurs différentes. Chacune des lignes commence et se termine avec un et comme pour signaler leur infinité. Walid Raad, toujours par la voix de Carlos Chahine, soutient que les artistes du futur lui ont communiqué ces noms, ceux d'artistes du passé, par télépathie et qu'il veut les rendre publics afin que toutes les éventuelles erreurs de transcription puissent être corrigées, la télépathie n'étant pas une science exacte. Pourtant, il ne s'agit pas d'un geste qui vise à remémorer le passé, mais plutôt «à ressusciter des lignes, couleurs, contours ou formes ", comme le dit l'artiste lui-même. Le passé et le futur deviennent la matière même du présent. L'archivage documentaire quitte le domaine du seul discours pour devenir image. D'une métaphore à l'autre, les pièces de "Scratching on Things I Could Disavow: A History of Art in the Arab World» donnent corps au récit de la performance, en même temps que la performance alimente le contenu de ces mêmes pièces. Walid Raad affirme encore: "la plupart des travaux que j'ai produits pour cette exposition-performance prennent la forme d'espaces scéniques, d'œuvres et d'hologrammes tridimensionnels ». II ne s'agit pas d'une exposition, ou tout au moins pas que d'une exposition. Il y a bien une présentation d'objets d'art, mais lorsque le discours du performeur les relie, leur offrant un cadre de signification, c'est l'exposition elle-même qui fait œuvre, déployant la profondeur du discours de l'artiste. Une fois la performance finie, les œuvres regagnent leur autonomie et le spectateur peut les regarder avec un œil différent. Revoir les pièces de Walid Raad à la lumière de ses mots provoque une sensation étrange. C'est un peu comme si le spectateur avait rendezvous avec des acteurs de théâtre, après la représentation. Ces objets qui interrogeaient leur public et se présentaient sans explication, comme des personnages en quête d'auteur, semblent l'avoir finalement trouvé. Ils se montrent désormais denses de signification, en faisant sens en tant que groupe, mais aussi en tant qu'individualités.

\section{Irène Panzani}

